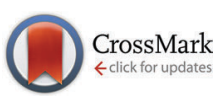

Cite this: Chem. Commun., 2016, 52,13179

DOI: $10.1039 / c 6 c c 90491 b$

www.rsc.org/chemcomm

\section{Correction: Proton conduction through oxygen functionalized few-layer graphene}

\author{
Chanderpratap Singh, ${ }^{a}$ Nikhil S., ${ }^{a}$ Anwesha Jana, ${ }^{a}$ Ashish Kumar Mishra* ${ }^{\mathrm{b}}$ and \\ Amit Paul*a
}

Correction for 'Proton conduction through oxygen functionalized few-layer graphene' by Chanderpratap Singh et al., Chem. Commun., 2016, 52, 12661-12664.

The authors regret that the values denoted on the $y$-axis of Fig. 3(d) are incorrect in the original article. A revised version of Fig. 3, in which the $y$-axis of Fig. 3(d) has been corrected, is included herein.
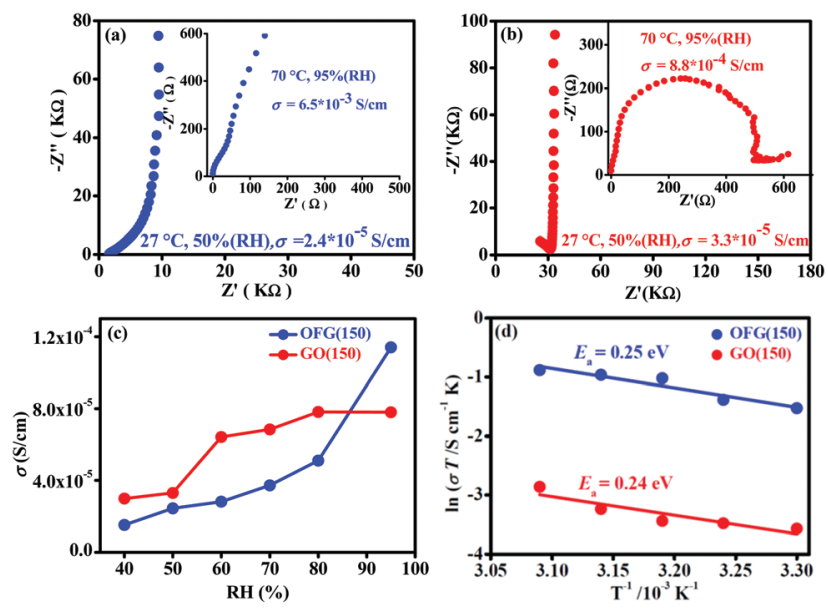

Fig. 3 Nyquist plots of (a) OFG(150) and (b) GO(150) with $\sigma$ values at a particular $\mathrm{RH}(\%)$ and temperature. (c) $\sigma$ vs. $\mathrm{RH}(\%)$ at $27{ }^{\circ} \mathrm{C}$ for $\mathrm{OFG}(150)$ and $\mathrm{GO}(150)$. (d) Plots of $\ln (\sigma T)$ vs. $T^{-1}$ for $\mathrm{OFG}(150)$ and $\mathrm{GO}(150)$ at $95 \% \mathrm{RH}$.

In addition to the above, the authors would also like to include the following text at the end of the paragraph beginning "Maier and Tuller highlighted the importance of..." on page 12664: "Besides, proton conduction in the bulk synthesized GO(150) is restricted due to turbostratic stacking of the layers, i.e. interruptions originating from interlayer interactions ${ }^{9}$ and such interruptions are not applicable for OFG(150). Finally, the higher $\mathrm{sp}^{2}$ carbon content of $\mathrm{OFG}(150)$ presumably enhances in-plane conduction compared to through-plane conduction, which also helps to achieve higher conductivity."

The Royal Society of Chemistry apologises for these errors and any consequent inconvenience to authors and readers.

\footnotetext{
${ }^{a}$ Department of Chemistry, Indian Institute of Science Education and Research (IISER) Bhopal, Madhya Pradesh, 462066, India. E-mail: apaul@iiserb.ac.in

${ }^{b}$ Department of Physics, Indian Institute of Science Education and Research (IISER) Bhopal, Madhya Pradesh, 462066, India. E-mail: akmishra@iiserb.ac.in
} 